\title{
The Best Path Analysis in Military Highway Transport Based on DEA and Multiobjective Fuzzy Decision-Making
}

\author{
Wu Juan, ${ }^{1,2}$ Lu Huapu, ${ }^{1}$ Sun Xu, ${ }^{1}$ Liu Xianfeng, ${ }^{2}$ and Yang Huijun ${ }^{2}$ \\ ${ }^{1}$ Institute of Transportation Engineering, Tsinghua University, Beijing 100084, China \\ ${ }^{2}$ Academy of Military Transportation, Tianjin 300161, China \\ Correspondence should be addressed to Wu Juan; wujuan_qh@yahoo.cn
}

Received 1 November 2013; Revised 6 January 2014; Accepted 8 January 2014; Published 20 February 2014

Academic Editor: Gang Chen

Copyright (c) $2014 \mathrm{Wu}$ Juan et al. This is an open access article distributed under the Creative Commons Attribution License, which permits unrestricted use, distribution, and reproduction in any medium, provided the original work is properly cited.

\begin{abstract}
Military transport path selection directly affects the transport speed, efficiency, and safety. To a certain degree, the results of the path selection determine success or failure of the war situation. The purpose of this paper is to propose a model based on DEA (data envelopment analysis) and multiobjective fuzzy decision-making for path selection. The path decision set is established according to a search algorithm based on overlapping section punishment. Considering the influence of various fuzzy factors, the model of optimal path is constructed based on DEA and multitarget fuzzy decision-making theory, where travel time, transport risk, quick response capability, and transport cost constitute the evaluation target set. A reasonable path set can be calculated and sorted according to the comprehensive scores of the paths. The numerical results show that the model and the related algorithms are effective for path selection of military transport.
\end{abstract}

\section{Introduction}

During the hi-tech local war, the mission of the military supplies support is very heavy, so the status of the military transport has become increasingly prominent [1]. Due to the characteristics of high flexibility and adaptability, military highway transport is still a significant mode of military transport [2]. Transport by car is an important means of conducting operational and tactical maneuver and supplies support $[3,4]$.

With the increasing uncertainty in occurrence and the quick decision of modern war, military transport needs more reliability and higher speed, so path optimization has become an important part of the military transport system. A good path selection plays an important role in completing the whole transport task on time, improving the efficiency of the transport, and enhancing logistic support capability.

There are many successful applications about routing optimization and selection in literatures [5-14]. However, in response to the antagonism and instability characteristics of modern war, to guarantee the stability and efficiency of the logistics, the wartime military highway transportation path selection should meet the following principles.
(1) Path must be feasible and better controlled traffic roads are required to be chosen in the first place to ensure that troops and supplies can reach the destination on time and safely.

(2) Try to select the path with short travel time, high safety, high economic benefit, and the least number of transfers as much as possible.

(3) The emergency transport plans are required to deal with various potential damage states to ensure that alternate routes may be selected flexibly after the enemy fire.

In view of the peculiarity of military highway transport in war, various factors should be comprehensively considered in path selection, such as the mileage of the path, speed, travel time, reliability, security, fuel consumption, transport loss, and supporting capability [1-4]. Some of these factors are fuzzy, uncertain, and difficult to quantify. Nowadays, most of the algorithms of path selection in military highway transport are based on the optimization of urban traffic, without considering the specific requirements of the military maneuvers and the fuzziness of these factors [15]. In this 
paper, to solve the path selection problem under the influence of various fuzzy factors in military transport, a model based on DEA and multiobjective fuzzy decision-making method is proposed.

\section{Path Decision Set Establishment}

For military transport path selection, sections that do not conform to the requirements of the transport task should be removed from the existing road network. After that, considering the particularity of military transportation, we also want to choose paths with high dissimilarity so as to equally distribute the risk as much as possible $[16,17]$.

To meet needs of warfare, transport task needs to be accomplished within the prescribed period of time. So in this paper, dissimilar paths [18-20] that are in accord with the time constraints are chosen as path optimization decision set.

Given a graph $G=(N, A, T)$, where $N=\{1,2, \ldots, n\}, A$ is the set of directed links (arcs) and $T$ is the set of time cost of each arc. Each $\operatorname{arc}(i, j)$ in the graph has a time cost $t_{i j}$.

The problem of searching the dissimilar path set $R_{0}$ from the source node to the sink node could be stated as follows:

$$
\begin{aligned}
& R_{0}=\left\{R \mid T(R) \leq T_{\max }\right\} \\
& \text { s.t. } \\
& T(R)=\sum_{(i, j) \in A} t_{i j} x_{i j} \\
& x_{i j}= \begin{cases}1 & \text { if }(i, j) \text { is in path } R ; \\
0 & \text { otherwise, }\end{cases}
\end{aligned}
$$

where $T_{\max }$ is the acceptable longest time to complete transport task; $T(R)$ is travel time of path $R$ from the source node to the sink node.

Using the dissimilar path algorithm based on overlapping punishment, the dissimilar path set $R_{0}$ consisting of $M$ paths that is in accord with the time constraints is obtained. The search algorithm consists of the following steps.

Step 1 (initialization). Consider

$$
m=0, \quad i=1, \quad R_{0}=\emptyset .
$$

Step 2. Using the Dijkstra algorithm [21] to search the shortest path $R_{i}$ from starting point to end point based on time cost weight, travel time on path $R_{i}$ is $T_{i}$.

Step 3. If $T_{i} \leq T_{\max }$ and $m<M$, go to the next step. Otherwise calculation stops.

Step 4. If $R_{i} \notin R_{0}$, update $m=m+1$ and $R_{0}=R_{0} \cup\left\{R_{i}\right\}$; go to the next step.

Step 5. Update $i=i+1$. Update travel time on each section as follows:

$$
t_{e}^{i}=\left(1+\frac{\alpha}{T_{\min }}\right)^{n} t_{e}^{0},
$$

where $t_{e}^{0}$ is the initial time weight of section $e, t_{e}^{i}$ is the time weight of section $e$ at $i$ loop, $\alpha$ is overlapping penalty coefficient, and $n$ is the used times of section $e$. Return to Step 2.

Using this algorithm, path decision set is obtained and arranged in order to increase travel time on each path:

$$
R_{0}=\left\{R_{1}, R_{2}, \ldots, R_{M}\right\}
$$

\section{Military Path Analysis Based on DEA and Multiobjective Fuzzy Decision-Making}

In the path selection of military transport, considering the influence of various fuzzy factors, multiobjective fuzzy decision-making method is an effective way [22-25].

3.1. Objective Factors of Path Selection. In wartime path selection, the decision-maker often considers simultaneously multiple conflicting objectives [26]. In this paper, travel time, transport risks, rapid response capability, and transport costs are selected as the objective factors.

(1) Travel Time. In the process of troops maneuvering, especially long-range maneuvering, most of the time was spent in transport. In order to shorten the whole troops maneuvering time, the travel time is an important factor to measure the vehicle turn-round efficiency.

(2) Transport Risk. During the war, supply lines and transportation facilities are often the prime targets of enemy fire. To ensure personnel and supplies reach their destination safely, transport risk will be an important factor to consider in path selection.

(3) Rapid Response Capability. In order to ensure the reliability and stability of military transport system after enemy attack, rapid response capability is required in wartime transport. There are various methods to enhance rapid response capabilities before the war, such as perfecting defense system of the critical traffic facilities in advance and putting forward the emergency transport plans to deal with various potential damage states. With these methods, alternate routes may be selected flexibly after the enemy fire.

(4) Transport Cost. When meeting the requirements of military demands, much more economic benefits could be pursued in path optimization. In the military transport, transport cost includes two aspects: one is traditional fuel cost, vehicle depreciation cost, and so forth; the other is the integrated support costs such as oil support and alert support.

\subsection{Membership Functions for Objective Factors}

3.2.1. Membership Functions for Travel Time. The purpose of transport time evaluation is only to compare the relative length of travel time on different routes. Therefore, 
the difference between travel time on each route and the shortest travel time is used as the evaluation criteria. Consider

$$
\Delta T=T-T_{\min }
$$

where $T_{\text {min }}$ is the shortest travel time on routes in $R_{0}$ and $\Delta T$ is travel time difference with the shortest travel time of each route. The smaller the value of $\Delta T$ is, the better this route is.

$$
\begin{aligned}
& \text { Define travel time remark set: } V_{t} \\
& \quad=\{\text { excellent, good, medium, poor }\}
\end{aligned}
$$

According to the experience of military transport, in general, for $\Delta T$, a value within the range $\left\{0 \sim 0.15 T_{\min }\right\}$ can be considered excellent; a larger value within the range $\left\{0.15 T_{\min } \sim 0.35 T_{\min }\right\}$ is good and one within $\left\{0.35 T_{\min } \sim\right.$ $\left.0.65 T_{\min }\right\}$ is medium; a value over 0.65 is always considered poor.

The common forms of membership functions include triangle, trapezoid, Gaussian, and clock form. Here, triangular fuzzy function is used to determine the membership degree of travel time. Then, membership functions of travel time difference $\Delta T$ on route $k$ are defined as follows [27]:

$$
\begin{aligned}
& U_{1 k}^{t}(\Delta t)= \begin{cases}1-\frac{10 \Delta T}{\left(3 T_{\text {min }}\right)} & 0 \leq \frac{\Delta T}{T_{\text {min }}}<0.15 \\
0 & \frac{\Delta T}{T_{\text {min }}} \geq 0.15\end{cases} \\
& U_{2 k}^{t}(\Delta t)= \begin{cases}\frac{10 \Delta T}{\left(3 T_{\text {min }}\right)} & 0 \leq \frac{\Delta T}{T_{\text {min }}}<0.15 \\
1.75-\frac{5 \Delta T}{T_{\text {min }}} & 0.15 \leq \frac{\Delta T}{T_{\text {min }}}<0.35 \\
0 & \frac{\Delta T}{T_{\text {min }}} \geq 0.35,\end{cases} \\
& U_{3 k}^{t}(\Delta t)= \begin{cases}0 & 0 \leq \frac{\Delta T}{T_{\text {min }}}<0.15 \\
-0.75+\frac{5 \Delta T}{T_{\text {min }}} & 0.15 \leq \frac{\Delta T}{T_{\text {min }}}<0.35 \\
2.25-\frac{10 \Delta T}{\left(3 T_{\text {min }}\right)} & 0.35 \leq \frac{\Delta T}{T_{\text {min }}}<0.65 \\
0 & \frac{\Delta T}{T_{\text {min }}} \geq 0.65,\end{cases} \\
& U_{4 k}^{t}(\Delta t)= \begin{cases}0 & 0 \leq \frac{\Delta T}{T_{\text {min }}}<0.35 \\
-1.25+\frac{10 \Delta T}{\left(3 T_{\text {min }}\right)} & 0.35 \leq \frac{\Delta T}{T_{\text {min }}}<0.65 \\
1 & \frac{\Delta T}{T_{\text {min }}} \geq 0.65 .\end{cases}
\end{aligned}
$$

So membership vector of travel time difference $\Delta T$ of route $k$ is

$$
U_{k}^{t}=\left(U_{1 k}^{t}(\Delta t), U_{2 k}^{t}(\Delta t), U_{3 k}^{t}(\Delta t), U_{4 k}^{t}(\Delta t)\right)^{T} .
$$

3.2.2. Membership Functions for Transport Risk, Rapid Response Capability, and Transport Cost. The three factors are of great fuzziness and difficult to quantify, and their membership functions are difficult to be defined directly. In this paper, comprehensive weighted statistics method is used to establish these membership functions.

(1) Define Remark Set for the Three Factors. Consider

$$
\begin{aligned}
& V_{s}=\{\text { excellent, good, medium, poor }\}, \\
& V_{r}=\{\text { excellent, good, medium, poor }\}, \\
& V_{c}=\{\text { excellent, good, medium, poor }\},
\end{aligned}
$$

where $V_{s}, V_{r}$, and $V_{c}$ are the remark sets of transport risk, rapid response capability, and transport cost.

(2) Determine Evaluation Personnel and the Weight of Each Type of Personnel. Based on the experience, evaluation personnel were selected from combat commanders, combat service staff officers, and transport professionals.

The weight of each type of personnel for transport risks assessment is

$$
R^{s}=\left(r_{1}^{s}, r_{2}^{s}, r_{3}^{s}\right)^{T}, \quad \text { where } \sum_{i=1}^{3} r_{i}^{s}=1, r_{i}^{s}>0 .
$$

The weight of each type of personnel for rapid response capability assessment is

$$
R^{r}=\left(r_{1}^{r}, r_{2}^{r}, r_{3}^{r}\right)^{T}, \quad \text { where } \sum_{i=1}^{3} r_{i}^{r}=1, r_{i}^{r}>0 .
$$

The weight of each type of personnel for transport costs assessment is

$$
R^{c}=\left(r_{1}^{c}, r_{2}^{c}, r_{3}^{c}\right)^{T}, \quad \text { where } \sum_{i=1}^{3} r_{i}^{c}=1, r_{i}^{c}>0 .
$$

(3) Membership Degree Calculation. Suppose type $i$ personnel have $n_{i}$ individuals, of whom $m_{j i k}$ individuals agree that route $k$ belongs to remark. Then, the evaluation results are as follows:

$$
A_{j i k}^{s}=\frac{m_{j i k}^{s}}{n_{i}}, \quad A_{j i k}^{r}=\frac{m_{j i k}^{r}}{n_{i}}, \quad A_{j i k}^{c}=\frac{m_{j i k}^{c}}{n_{i}} .
$$
follows:

Then, membership degree of each objective factor is as

$$
\begin{aligned}
& U_{j k}^{s}=\left(A_{j 1 k}^{s}, A_{j 2 k}^{s}, A_{j 3 k}^{s}\right) \times R^{s}, \\
& U_{j k}^{r}=\left(A_{j 1 k}^{r}, A_{j 2 k}^{r}, A_{j 3 k}^{r}\right) \times R^{r}, \\
& U_{j k}^{c}=\left(A_{j 1 k}^{c}, A_{j 2 k}^{c}, A_{j 3 k}^{c}\right) \times R^{c},
\end{aligned}
$$

where $i=1,2,3 ; j=1,2,3,4 ; k=1,2,3, \ldots, M$.

Then, the evaluation vector of route $k$ was obtained as follows:

$$
\begin{aligned}
& U_{k}^{s}=\left(U_{1 k}^{s}, U_{2 k}^{s}, U_{3 k}^{s}, U_{4 k}^{s}\right)^{T}, \\
& U_{k}^{r}=\left(U_{1 k}^{r}, U_{2 k}^{r}, U_{3 k}^{r}, U_{4 k}^{r}\right)^{T}, \\
& U_{k}^{c}=\left(U_{1 k}^{c}, U_{2 k}^{c}, U_{3 k}^{c}, U_{4 k}^{c}\right)^{T} .
\end{aligned}
$$


Then, the evaluation matrix of route set $R_{0}$ was obtained as follows:

$$
\begin{aligned}
& U^{s}=\left(U_{1}^{s}, U_{2}^{s}, U_{3}^{s}, U_{4}^{s}\right)^{T}, \\
& U^{r}=\left(U_{1}^{r}, U_{2}^{r}, U_{3}^{r}, U_{4}^{r}\right)^{T}, \\
& U^{c}=\left(U_{1}^{c}, U_{2}^{c}, U_{3}^{c}, U_{4}^{c}\right)^{T} .
\end{aligned}
$$

3.3. DEA Model for Evaluation. DEA method [28-30] is a sort of evaluating method used to evaluate the relative validity of decision-making unit with multiple inputs and outputs. In DEA method, $C^{2} R$ model is the earliest one and most widely used now. So the following evaluation is mainly according to $C^{2} R$ model.

(1) Using path from path decision set as decision-making unit and using membership degree as input and output indicators, for each path, the score value of each remark grade on each objective factor is calculated.

In this paper, $V=$ \{excellent, good, medium, poor $\}$; the corresponding membership degree of "medium" and "poor" is used as input and the corresponding membership degree of "excellent" and "good" is used as output.

The weight vectors of input and output are given as the variable vectors as follows:

$$
\vec{v}=\left(v_{1}, \ldots, v_{m}\right)^{T}, \quad \vec{u}=\left(u_{1}, \ldots, u_{s}\right)^{T} .
$$

For route $k$, the score value vector on each objective factor can be calculated as follows:

$$
h_{k}=\frac{\vec{u}^{T} \vec{y}_{k}}{\vec{v}^{T} \vec{x}_{k}}=\frac{\sum_{i=1}^{s} u_{i} y_{i k}}{\sum_{j=1}^{m} v_{j} x_{j k}}, \quad k=1, \ldots, M .
$$

By choosing appropriate weight vector $\vec{\mu}$ and $\vec{v}$ to maximize the value of $h_{k}$, the optimal score of route $k$ on this objective factor can be obtained. For example, for route $k_{0}$, $C^{2} R$ model is proposed as follows:

$$
\begin{array}{ll}
\max & h_{k 0}=\frac{\sum_{i=1}^{s} u_{i} y_{i k 0}}{\sum_{j=1}^{m} v_{j} x_{j k 0}} \\
\text { s.t. } & \frac{\sum_{i=1}^{s} u_{i} y_{i k}}{\sum_{j=1}^{m} v_{j} x_{j k}} \leq 1 \quad k=1, \ldots, M, \\
& u_{i} \geq 0, \quad i=1, \ldots, s, \\
& v_{j} \geq 0, \quad j=1, \ldots, m .
\end{array}
$$

This model is fractional program and can be converted into linear program to solve by using the Charnes-Cooper transformation.

Taking membership degree of route $k$ into the model, the score value $\theta_{k i}$ on each objective factor can be calculated.

(2) Using path from path decision set as decision-making unit and using $\theta_{k i}$ as input and output indicators, the final score of each path can be calculated. The path with the highest score is the optimal one.
TABLE 1: Road section travel time.

\begin{tabular}{lccccccccccc}
\hline Section & $1-2$ & $1-3$ & $1-4$ & $2-3$ & $2-5$ & $2-6$ & $3-4$ & $4-6$ & $5-6$ & $5-7$ & $6-7$ \\
Travel time (h) & 1 & 2 & 1 & 1 & 5 & 6 & 3 & 5 & 4 & 4 & 5 \\
\hline
\end{tabular}

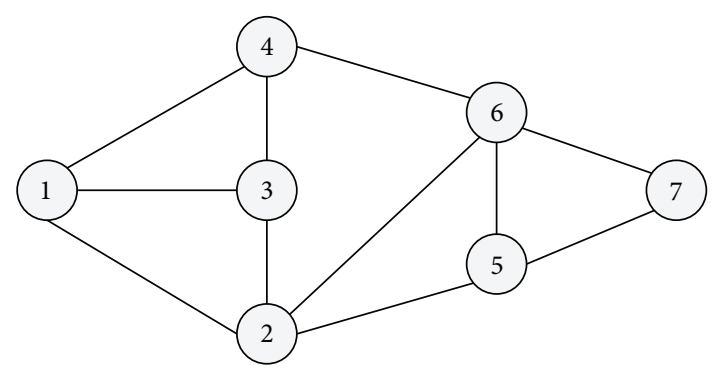

FIGURE 1: The road networks.

\section{Case Studies}

To meet the demand of the combat replenishment, a batch of military supplies need to be moved from node 1 to node 7 in time. Figure 1 shows the layout of the road network. Travel time on each road section is shown in Table 1. According to the requirements of the combat process, this transport task is required to be completed within $15 \mathrm{~h}$ and 5 alternative paths need to be selected for safety reasons.

(1) Dissimilar Path Set Calculation. Using the Dijkstra algorithm to search the shortest path $R_{1}$ based on time weight, travel time on path $R_{1}$ is $T_{1}$. The other four paths are obtained by using the dissimilar path algorithm based on overlapping punishment. Suppose that overlapping penalty coefficient is 0.5 ; the results are:

$$
\begin{gathered}
R_{1}=\{1,2,5,7\}, \quad R_{2}=\{1,4,6,7\}, \quad R_{3}=\{1,3,2,5,7\}, \\
R_{4}=\{1,2,6,7\}, \quad R_{5}=\{1,4,6,5,7\} .
\end{gathered}
$$

Path decision set is $R_{0}=\left\{R_{1}, R_{2}, R_{3}, R_{4}, R_{5}\right\}$ and the corresponding travel time set is $T_{0}=\{10,11,12,12,14\}$. The shortest travel time $T_{\min }=10$.

(2) Membership Degree Calculation. Evaluation personnel consist of 5 battle commanders, 10 combat service staff officers, and 15 transportation professionals.

The weight of each type of personnel for transport risk assessment is $R_{s}=(0.45,0.35,0.2)^{T}$.

The weight of each type of personnel for rapid response capability is $R_{r}=(0.4,0.35,0.25)^{T}$.

The weight of each type of personnel for transport cost is $R_{c}=(0.2,0.3,0.5)^{T}$.

The evaluation results for path decision set by the evaluation personnel are shown in Table 2. Each element of the evaluation vector represents the number of each type of evaluation personnel who agree with this grade.

The difference between travel time on each route and the shortest travel time is shown in Table 3. 
TABLE 2: Evaluation results by evaluation personnel.

\begin{tabular}{|c|c|c|c|c|c|c|}
\hline & Path & $R_{1}$ & $R_{2}$ & $R_{3}$ & $R_{4}$ & $R_{5}$ \\
\hline \multirow{4}{*}{ 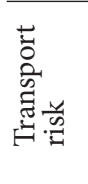 } & Excellent & $(0,2,4)$ & $(1,3,5)$ & $(2,5,7)$ & $(3,6,8)$ & $(3,4,7)$ \\
\hline & Good & $(2,3,5)$ & $(2,4,5)$ & $(2,4,5)$ & $(2,3,5)$ & $(2,4,6)$ \\
\hline & Medium & $(2,4,5)$ & $(2,2,4)$ & $(1,1,3)$ & $(0,1,2)$ & $(0,2,2)$ \\
\hline & Poor & $(1,1,1)$ & $(0,1,1)$ & $(0,0,0)$ & $(0,0,0)$ & $(0,0,0)$ \\
\hline \multirow{4}{*}{ 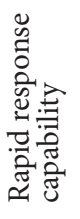 } & Excellent & $(2,3,5)$ & $(2,4,5)$ & $(1,4,5)$ & $(2,4,6)$ & $(1,3,5)$ \\
\hline & Good & $(2,4,5)$ & $(1,2,4)$ & $(2,5,6)$ & $(2,3,4)$ & $(3,6,4)$ \\
\hline & Medium & $(1,2,3)$ & $(2,3,5)$ & $(1,1,3)$ & $(1,2,3)$ & $(1,1,4)$ \\
\hline & Poor & $(0,1,2)$ & $(0,1,1)$ & $(1,0,1)$ & $(0,1,2)$ & $(0,0,2)$ \\
\hline \multirow{4}{*}{ 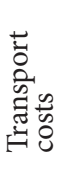 } & Excellent & $(3,5,7)$ & $(2,4,7)$ & $(1,4,6)$ & $(2,5,6)$ & $(1,3,4)$ \\
\hline & Good & $(2,4,5)$ & $(2,3,5)$ & $(2,4,5)$ & $(2,3,7)$ & $(1,2,4)$ \\
\hline & Medium & $(0,1,3)$ & $(1,3,2)$ & $(2,1,3)$ & $(1,2,1)$ & $(2,3,4)$ \\
\hline & Poor & $(0,0,0)$ & $(0,0,1)$ & $(0,1,1)$ & $(0,0,1)$ & $(1,2,3)$ \\
\hline
\end{tabular}

TABLE 3: Travel time difference of each route.

\begin{tabular}{lccccc}
\hline Path & $R_{1}$ & $R_{2}$ & $R_{3}$ & $R_{4}$ & $R_{5}$ \\
Travel time difference & 0 & 1 & 2 & 2 & 4 \\
\hline
\end{tabular}

TABLE 4: Membership degree.

\begin{tabular}{|c|c|c|c|c|c|c|}
\hline & Route & $R_{1}$ & $R_{2}$ & $R_{3}$ & $R_{4}$ & $R_{5}$ \\
\hline \multirow{4}{*}{ 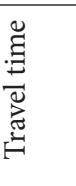 } & Excellent & 1 & 0.667 & 0 & 0 & 0 \\
\hline & Good & 0 & 0.333 & 0.75 & 0.75 & 0 \\
\hline & Medium & 0 & 0 & 0.25 & 0.25 & 0.917 \\
\hline & Poor & 0 & 0 & 0 & 0 & 0.083 \\
\hline \multirow{4}{*}{ 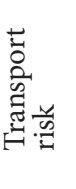 } & Excellent & 0.123 & 0.262 & 0.448 & 0.587 & 0.503 \\
\hline & Good & 0.352 & 0.387 & 0.387 & 0.352 & 0.400 \\
\hline & Medium & 0.387 & 0.303 & 0.165 & 0.061 & 0.097 \\
\hline & Poor & 0.138 & 0.048 & 0.0 & 0.0 & 0.0 \\
\hline \multirow{4}{*}{ 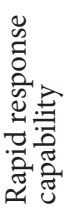 } & Excellent & 0.348 & 0.384 & 0.304 & 0.400 & 0.268 \\
\hline & Good & 0.384 & 0.217 & 0.435 & 0.332 & 0.517 \\
\hline & Medium & 0.200 & 0.348 & 0.165 & 0.200 & 0.182 \\
\hline & Poor & 0.068 & 0.051 & 0.096 & 0.068 & 0.033 \\
\hline \multirow{4}{*}{ 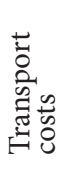 } & Excellent & 0.503 & 0.433 & 0.360 & 0.430 & 0.263 \\
\hline & Good & 0.367 & 0.337 & 0.367 & 0.404 & 0.234 \\
\hline & Medium & 0.130 & 0.197 & 0.210 & 0.133 & 0.303 \\
\hline & Poor & 0.0 & 0.033 & 0.063 & 0.033 & 0.200 \\
\hline
\end{tabular}

TABLE 5: Score value of each route on each remark grade.

\begin{tabular}{lccccc}
\hline Remark grade & $R_{1}$ & $R_{2}$ & $R_{3}$ & $R_{4}$ & $R_{5}$ \\
\hline Excellent & 0.551 & 0.462 & 0.245 & 0.313 & 0.232 \\
Good & 0.238 & 0.324 & 0.519 & 0.498 & 0.250 \\
Medium & 0.163 & 0.185 & 0.204 & 0.169 & 0.442 \\
Poor & 0.048 & 0.029 & 0.032 & 0.020 & 0.076 \\
\hline
\end{tabular}

TABLE 6: Final score of each path.

\begin{tabular}{lccccc}
\hline Route & $R_{1}$ & $R_{2}$ & $R_{3}$ & $R_{4}$ & $R_{5}$ \\
Final score & 0.393 & 0.371 & 0.312 & 0.340 & 0.250 \\
\hline
\end{tabular}

Membership degree is calculated in Table 4.

(3) Comprehensive Evaluation. Taking membership degree of route $k$ into DEA model (8), the score value $\theta_{k i}$ on each objective factor can be calculated as shown in Table 5 .

Using $\theta_{k i}$ as input and output indicators, the final score of each path can be calculated as shown in Table 6 .

So, the calculation results are sorted in a descending order: $R_{1}>R_{2}>R_{4}>R_{3}>R_{5}$.

\section{Conclusions}

In this paper, path selection problem considering the influence of various fuzzy factors in military transport is investigated. Based on overlapping section punishment search algorithm, the path decision set is established. Then, a path selection decision model is presented based on DEA and multiobjective fuzzy decision-making method, where the evaluation target set consists of travel time, transport risk, quick response capability, and transport cost. For factors of great fuzziness and difficult to quantify, comprehensive weighted statistics method is used to establish the membership functions. Using path as decision-making unit, the comprehensive evaluation by DEA model can sort the path set, where the path with the higher comprehensive score indicates the much better one. Finally, the calculation results show the validity and effectiveness of the proposed method.

\section{Conflict of Interests}

The authors declare that there is no conflict of interests regarding the publication of this paper.

\section{Acknowledgment}

This work is partly supported by Science and Technology Program of Beijing, China (Grant no. Z121100000312101).

\section{References}

[1] X. D. Sun, Military Logistics, PLA Publishing Press, Beijing, China, 2002.

[2] Z. R. Chen and P. X. Bao, Military Transportation, PLA Publishing Press, Beijing, China, 2001.

[3] X. D. Luo, Combat Power Projection, Military Science Press, Beijing, China, 2003.

[4] S. S. Yu and G. H. Yu, Force Projection, National Defense University, Beijing, China, 2003.

[5] B. Y. Chen, W. H. K. Lam, A. Sumalee, and Z.-L. Li, "Reliable shortest path finding in stochastic networks with spatial correlated link travel times," International Journal of Geographical Information Science, vol. 26, no. 2, pp. 365-386, 2012.

[6] B. Y. Chen, W. H. K. Lam, A. Sumalee, Q. Q. Li, H. Shao, and Z. $\mathrm{X}$. Fang, "Finding reliable shortest paths in road networks under 
uncertainty," Networks \& Spatial Economics, vol. 13, pp. 123-148, 2013.

[7] B. Y. Chen, W. H. K. Lam, Q. Q. Li, A. Sumalee, and K. Yan, "Shortest path finding problem in stochastic time-dependent road networks with stochastic first-in-first-out property," IEEE Transactions on Intelligent Transportation Systems, vol. 14, no. 4, pp. 1907-1917, 2013.

[8] B. Z. Yao, P. Hu, M. H. Zhang, and S. Wang, "Artificial bee colony algorithm with scanning strategy for periodic vehicle routing problem," SIMULATION, vol. 89, no. 6, pp. 762-770, 2013.

[9] B. Yu, W. H. K. Lam, and M. L. Tam, "Bus arrival time prediction at bus stop with multiple routes," Transportation Research C, vol. 19, no. 6, pp. 1157-1170, 2011.

[10] B. Yu and Z. Z. Yang, "An ant colony optimization model: the period vehicle routing problem with time windows," Transportation Research E, vol. 47, no. 2, pp. 166-181, 2011.

[11] B. Yu, Z. Z. Yang, and L. I. S, "Real-time partway deadheading strategy based on transit service reliability assessment," Transportation Research A, vol. 46, no. 8, pp. 1265-1279, 2012.

[12] B. Yu, Z.-Z. Yang, and J.-X. Xie, "A parallel improved ant colony optimization for multi-depot vehicle routing problem," Journal of the Operational Research Society, vol. 62, no. 1, pp. 183-188, 2011.

[13] B. Yu, Z.-Z. Yang, and B. Yao, "An improved ant colony optimization for vehicle routing problem," European Journal of Operational Research, vol. 196, no. 1, pp. 171-176, 2009.

[14] B. Z. Yao, P. Hu, X. H. Lu, J. J. Gao, and M. H. Zhang, "Transit network design based on travel time reliability," Transportation Research C. In press.

[15] Y. F. Shi, Optimizing transportation route with uncertain factor in war [Ph.D. thesis], Southeast Jiaotong University, 2005.

[16] I. Akgün and B. Ç. Tansel, "Optimization of transportation requirements in the deployment of military units," Computers and Operations Research, vol. 34, no. 4, pp. 1158-1176, 2007.

[17] K. McKinzie and J. W. Barnes, "A review of strategic mobility models supporting the defense transportation system," Mathematical and Computer Modelling, vol. 39, no. 6-8, pp. 839-868, 2004.

[18] V. Akgün, E. Erkut, and R. Batta, "On finding dissimilar paths," European Journal of Operational Research, vol. 121, no. 2, pp. 232-246, 2000.

[19] P. Dell'Olmo, M. Gentili, and A. Scozzari, "On finding dissimilar Pareto-optimal paths," European Journal of Operational Research, vol. 22, no. 12, pp. 106-109, 2005.

[20] M. Caramia, S. Giordani, and A. Iovanella, "On the selection of $\mathrm{k}$ routes in multiobjective hazmat route planning," IMA Journal Management Mathematics, vol. 21, no. 3, pp. 239-251, 2010.

[21] S. D. Gu, Operational Research, Tsinghua University Press, Beijing, China, 2005.

[22] A. Quattrone and A. Vitetta, "Random and fuzzy utility models for road route choice," Transportation Research E, vol. 47, no. 6, pp. 1126-1139, 2011.

[23] Y. J. Liu and K. F. Yan, "Fuzzy multi-criteria decision-making method in planning of transport hub," Journal of Wuhan Urban Construction Institute, vol. 23, no. 2, pp. 63-66, 2006.

[24] W. K. M. Brauers, E. K. Zavadskas, F. Peldschus, and Z. Turskis, "Multi-objective decision-making for road design," Transport, vol. 23, no. 3, pp. 183-193, 2008.

[25] D. D. Wu, Y. Zhang, D. Wu, and D. L. Olson, "Fuzzy multiobjective programming for supplier selection and risk modeling: a possibility approach," European Journal of Operational Research, vol. 200, no. 3, pp. 774-787, 2010.
[26] Y. Liu, M. P. Yun, and G. X. Peng, "A multi-objective programming model of route choice of emergency vehicles before travel," Journal of Highway and Transportation Research and Development, vol. 26, no. 8, pp. 135-139, 2009.

[27] Q. C. Wu, T. Z. Chen, and Y. Su, "The best path analysis based on multi-target fuzzy decision-making," Journal of Computer Simulation, vol. 32, no. 18, pp. 156-160, 2010.

[28] F. H. Lotfi, G. R. Jahanshahloo, M. Khodabakhshi, M. RostamyMalkhlifeh, Z. Moghaddas, and M. Vaez-Ghasemi, "A review of ranking models in data envelopment analysis," Journal of Applied Mathematics, vol. 2013, Article ID 492421, 20 pages, 2013.

[29] W.-W. Fu and W.-Z. Jin, "Fuzzy comprehensive evaluation of urban public transport system based on DEA model," Journal of Wuhan University of Technology, vol. 32, no. 18, pp. 156-160, 2010.

[30] M. Zerafat Angiz L., A. Emrouznejad, and A. Mustafa, "Fuzzy assessment of performance of a decision making units using DEA: a non-radial approach," Expert Systems with Applications, vol. 37, no. 7, pp. 5153-5157, 2010. 


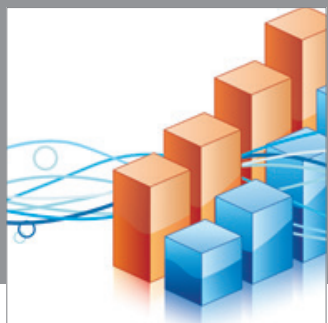

Advances in

Operations Research

mansans

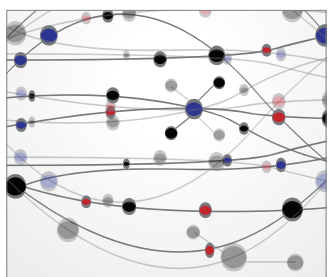

The Scientific World Journal
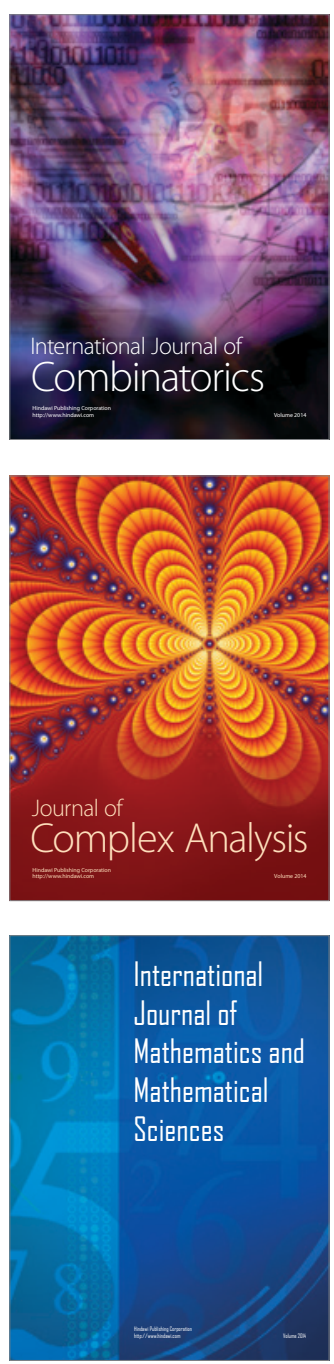
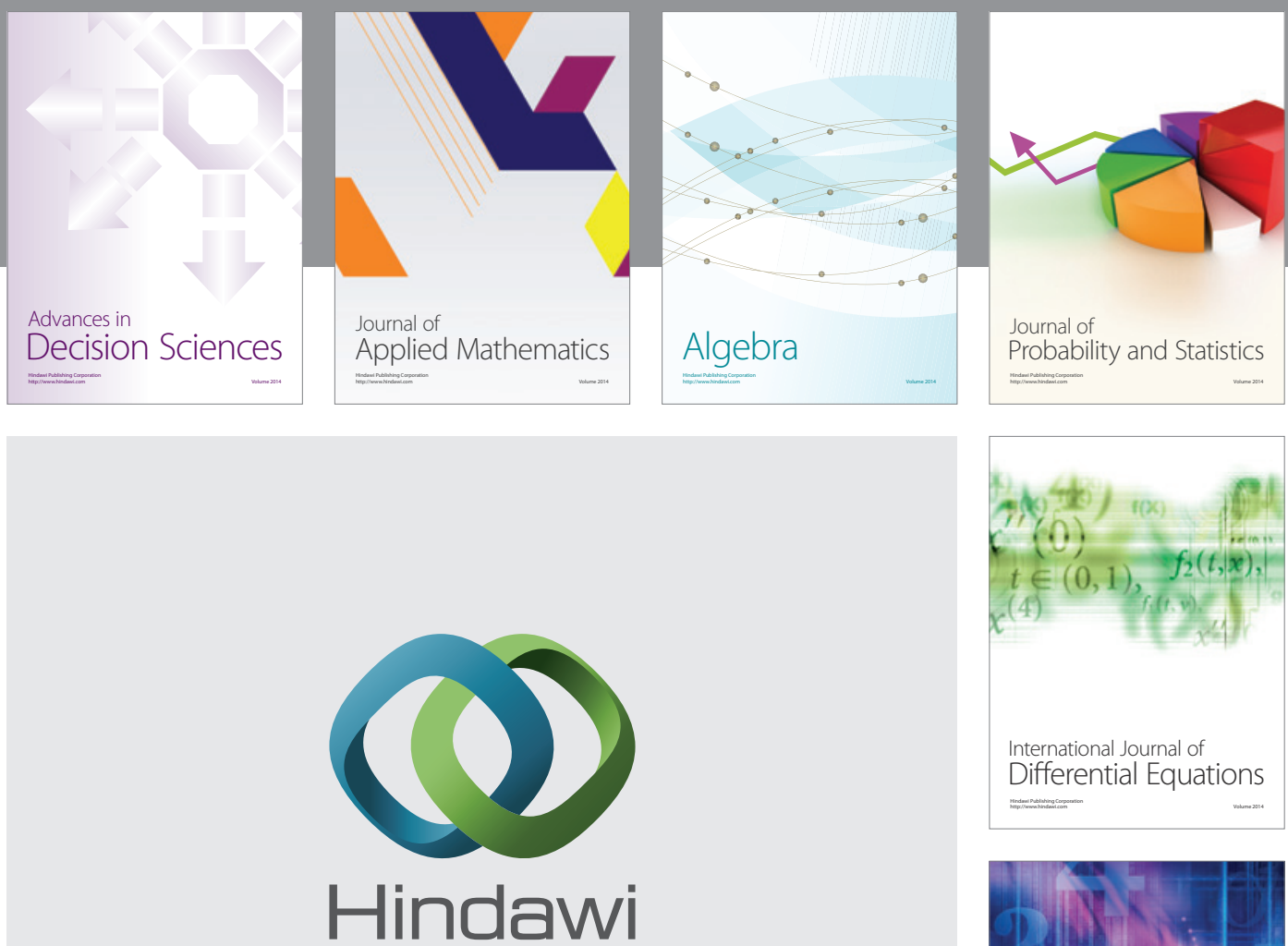

Submit your manuscripts at http://www.hindawi.com
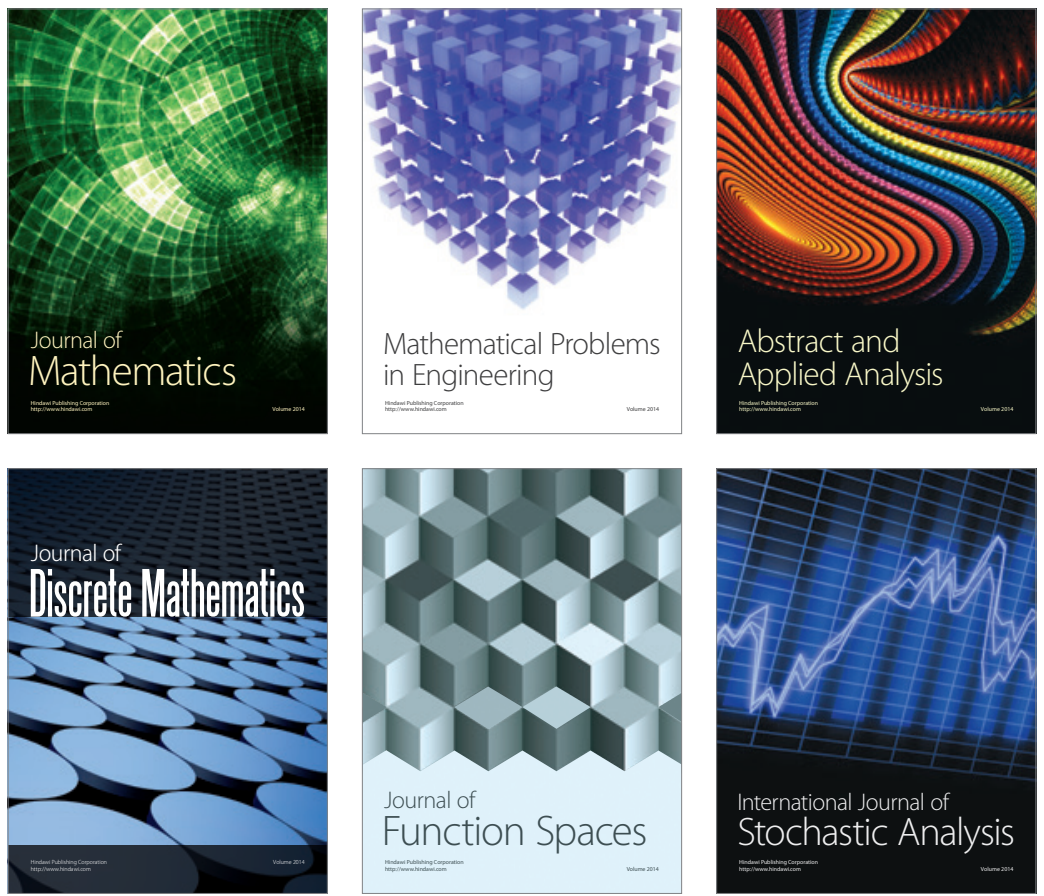

Journal of

Function Spaces

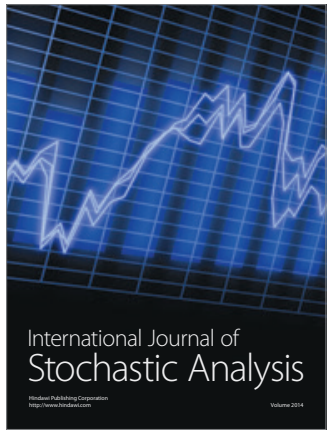

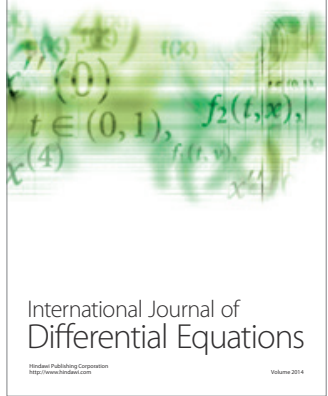
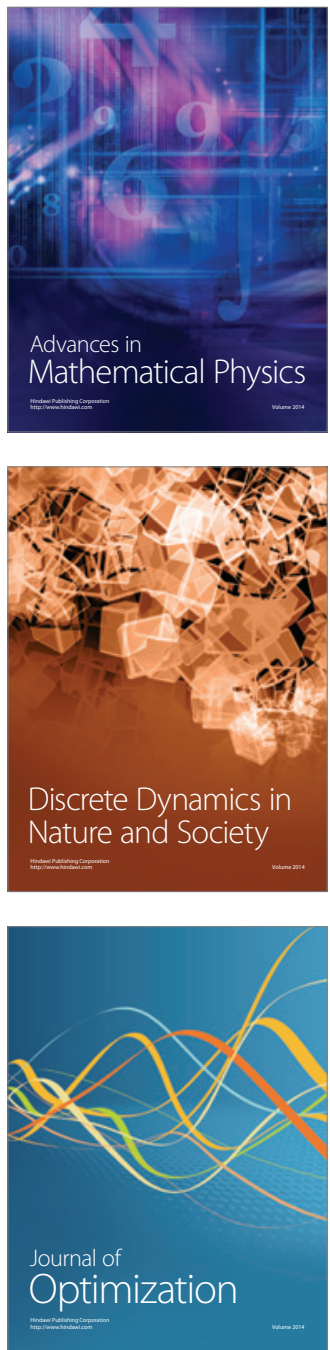